\title{
Pregnancy outcomes following botulinum toxin type A exposure: A systematic review
}

\author{
Noha Abdelkader, MSc ${ }^{1,3}$, Elif Keskin-Arslan ${ }^{1,2}$, M.D, Hilal Erol-Coskun ${ }^{1,2}$, M.D, Gözde
} Küçüksolak $^{1}$, S.M.S, Yusuf Cem Kaplan ${ }^{1,2}$, M.D.

1. Terafar - Izmir Katip Celebi University Teratology Information, Training and Research Center, Izmir, Turkey.

2. Izmir Katip Celebi University School of Medicine, Department of Pharmacology Izmir, Turkey.

3. Department of Clinical Pharmacy, Faculty of Pharmacy, Misr University for Science and Technology, Egypt.

Yusuf Cem Kaplan, M.D., Professor of Pharmacology

Director, Terafar, Izmir Katip Celebi University Teratology Information, Training and Research Center, Izmir, Turkey.

Address: Izmir Katip Celebi Ataturk Egitim ve Arastirma Hastanesi, Klinik Farmakoloji ve Toksikoloji Birimi, 35360, Karabaglar, Izmir, TURKEY.

\begin{abstract}
Objective: To evaluate the available human data to-date in order to assess whether the prenatal exposure to botulinum toxin type A (BTX-A) is associated with major congenital malformations and other adverse pregnancy outcomes.

Methods: Searches were conducted in PubMed/MEDLINE and Reprotox in November 2017. Cohort and case-control studies, case series, case reports were the primary data of interest.

Results: No controlled studies but case series and case reports of therapeutic BTX-A administration during pregnancy were identified. Case reports regarding pregnant women with botulism were also reviewed.

Conclusions: Limited data suggests that BTX-A exposure for therapeutic indications during pregnancy does not seem to be associated with an increase in risk of major congenital malformations. Rates of fetal loss were substantially different between prospective and retrospective data. Controlled epidemiological studies are needed to refute or support our findings.
\end{abstract}

Key Words: Botox; botulinum toxin; congenital abnormalities; onabotulinumtoxinA; pregnancy.

\section{Introduction}

Botulinum toxin is considered one of the most potent normally occurring toxin produced by Clostridium botulinum bacteria, which is able to block the neuromuscular transmission by binding to the motor or sympathetic nerve terminals and inhibiting the release of acetylcholine causing partial chemical denervation of the muscle resulting in a localized reduction in muscle activity [1-3]. Botulinum toxin has eight serotypes (A to $\mathrm{H}$ ), both type $A$ and $B$ are the only used in the clinical practice. Type A toxins are Botox ${ }^{\circledR}$ (onabotulinumtoxinA), Xeomin ${ }^{\circledR}$ (incobotulinumtoxinA), and Dysport ${ }^{\circledR}$ (abobotulinumtoxinA) while type 
B toxin is Myobloc ${ }^{\circledR}$ (rimabotulinumtoxinB) [1]. Botulinum toxin type A (BTX-A) has been used for injection in the treatment of several medical disorders and cosmetic purposes; strabismus, blepharospasm, hemifacial spasm (HFS) cervical dystonia (CD), hyperactive bladder, sialorrhea, hyperhidrosis, migraine, spasticity and to improve wrinkle appearance [1,3], while botulinum toxin type B (BTX-B) is usually spared for conditions which are refractory to BTX-A [4]. Given that approximately half of all pregnancies are unplanned and the increased use of BTX-A in the management of women of reproductive age, the incidental exposure of pregnant women to the drug became more common [5]. Hence the objective of this systematic review is to qualitatively evaluate the human data to date in order to assess whether the prenatal exposure to BTX-A is associated with any birth defects or other adverse pregnancy outcomes.

\section{Methods}

Search was conducted in PubMed/MEDLINE and Reprotox, in November 2017 by authors (EKA and HEC) using the search terms (botulinum toxin OR onabotulinumtoxinA) AND (pregnancy OR prenatal) in PubMed/MEDLINE and (botulinum toxin, onabotulinumtoxinA) in Reprotox. No language or date restrictions were applied. Hand searching of the reference lists of the relevant articles was also performed. The review following the Preferred Reporting Items for Systematic Reviews and Meta-analyses (PRISMA) statement [6]. Cohort and case-control studies, case series, case reports with prenatal exposure to botulinum toxin were the primary data of interest. Animal studies, editorials, and reviews were excluded.

\section{Results}

Thirty-two full-text papers were retrieved and screened for eligibility, PRISMA flow diagram details the search and selection process [Figure 1]. We were only able to retrieve case series and case reports, but no controlled epidemiological studies have been found. Nineteen studies are included in this review; 11 studies ( 1 case series [7], 1 survey [8] and 8 case reports [9-17]) for the therapeutic BTX-A (onabotulinumtoxinA) exposure during pregnancy included a total of 259 exposed women and 269 infants were with a known outcome (Table 1) and 8 studies (1 case series [18] and 7 case reports [19-25]) reporting botulism during pregnancy, included a total of 10 women (10 infants) [Table 2].

Table 1. Descriptive data of included studies for therapeutic exposure for Botulinum toxin A (BTX-A) during pregnancy

\begin{tabular}{|c|c|c|c|c|c|c|c|c|c|c|}
\hline Study & $\begin{array}{c}\text { Study } \\
\text { location }\end{array}$ & $\begin{array}{l}\text { Type of } \\
\text { Study }\end{array}$ & $\begin{array}{c}\text { No of } \\
\text { located } \\
\text { cases } \\
\text { (fetus no.) }\end{array}$ & $\begin{array}{l}\text { Women age } \\
\text { (cases no.) }\end{array}$ & $\begin{array}{l}\text { BTX-A } \\
\text { used }\end{array}$ & $\begin{array}{c}\text { Indication } \\
\text { for BTX- A } \\
\text { treatment } \\
\text { (cases no.) }\end{array}$ & $\begin{array}{c}\text { Dose of } \\
\text { BTX-A } \\
\text { (cases no.) }\end{array}$ & $\begin{array}{c}\text { Gestational } \\
\text { age at } \\
\text { BTX -A } \\
\text { exposure } \\
\text { ( cases no.) }\end{array}$ & $\begin{array}{l}\text { Other } \\
\text { drugs }\end{array}$ & $\begin{array}{l}\text { Pregnancy } \\
\text { outcome } \\
\text { (cases no. ) }\end{array}$ \\
\hline \multirow{4}{*}{$\begin{array}{c}\text { Newman et } \\
\text { al. } \\
\text { (2004) [11] }\end{array}$} & \multirow[t]{4}{*}{ USA } & \multirow[t]{4}{*}{ Case report } & $\begin{array}{l}1 \text { case }^{a} \\
\text { (4) }\end{array}$ & & & & & & & \\
\hline & & & $\begin{array}{c}\text { First } \\
\text { pregnancy }\end{array}$ & 26 years & $\begin{array}{l}\text { Onabotulinu } \\
\text { mtoxinA }\end{array}$ & $\begin{array}{l}\text { Cervical } \\
\text { dystonia }\end{array}$ & $\begin{array}{c}\text { Four } \\
\text { injections } \\
1200 \mathrm{U} \\
\text { With } 300 \mathrm{U} \\
\text { in each }\end{array}$ & $\begin{array}{l}3 \text { months } \\
\text { preconcepti } \\
\text { on, the first, } \\
\text { second and } \\
\text { third } \\
\text { trimester }\end{array}$ & $\begin{array}{c}\text { Trihexyphe } \\
\text { nidyl } \\
\text { Discontinue } \\
\text { d }\end{array}$ & $\begin{array}{c}\text { Healthy } \dagger \\
\text { newborn }\end{array}$ \\
\hline & & & $\begin{array}{c}\text { Second } \\
\text { pregnancy }\end{array}$ & 28 years & $\begin{array}{l}\text { Onabotulinu } \\
\text { mtoxinA }\end{array}$ & $\begin{array}{l}\text { Cervical } \\
\text { dystonia }\end{array}$ & $\begin{array}{c}\text { Three } \\
\text { injections } \\
900 \mathrm{U} \\
\text { With } 300 \mathrm{U} \\
\text { in each. }\end{array}$ & $\begin{array}{l}3 \text { months } \\
\text { preconcepti } \\
\text { on, the first } \\
\text { and third } \\
\text { trimester. }\end{array}$ & $\begin{array}{c}\text { Baclofen } \\
\text { Discontinue } \\
\text { d }\end{array}$ & $\begin{array}{l}\text { Healthy } \\
\text { newborn }\end{array}$ \\
\hline & & & $\begin{array}{c}\text { Third } \\
\text { Pregnancy }\end{array}$ & 30 years & $\begin{array}{l}\text { Onabotulinu } \\
\text { mtoxinA }\end{array}$ & $\begin{array}{l}\text { Cervical } \\
\text { dystonia }\end{array}$ & $\begin{array}{c}\text { Two } \\
\text { injections } \\
600 \mathrm{U}\end{array}$ & $\begin{array}{l}3 \text { months } \\
\text { pre- } \\
\text { conception }\end{array}$ & $\begin{array}{c}\text { Baclofen } \\
\text { Discontinue } \\
\text { d }\end{array}$ & $\begin{array}{l}\text { Healthy } \\
\text { newborn }\end{array}$ \\
\hline
\end{tabular}




\begin{tabular}{|c|c|c|c|c|c|c|c|c|c|c|}
\hline & & & & & & & $\begin{array}{l}\text { With } 300 \\
\mathrm{U} \text { in each. }\end{array}$ & $\begin{array}{l}\text { and the } \\
\text { second } \\
\text { trimester. }\end{array}$ & & \\
\hline & & & $\begin{array}{c}\text { Fourth } \\
\text { pregnancy }\end{array}$ & 32 years & $\begin{array}{l}\text { Onabotulinu } \\
\text { mtoxinA }\end{array}$ & $\begin{array}{l}\text { Cervical } \\
\text { dystonia }\end{array}$ & $\begin{array}{c}\text { Three } \\
\text { injections } \\
900 \mathrm{U} \\
\text { With } 300 \mathrm{U} \\
\text { in each }\end{array}$ & $\begin{array}{l}3 \text { months } \\
\text { preconcepti } \\
\text { on, the } \\
\text { second and } \\
\text { the third } \\
\text { trimester. }\end{array}$ & $\begin{array}{c}\text { Baclofen } \\
\text { Discontinue } \\
\text { d }\end{array}$ & $\begin{array}{l}\text { Healthy } \\
\text { newborn }\end{array}$ \\
\hline \multirow{3}{*}{$\begin{array}{c}\text { Bodkin et } \\
\text { al. }(2005) \\
{[12]}\end{array}$} & \multirow[t]{3}{*}{ USA } & Case report & 2 cases & & & & & & & \\
\hline & & & Case 1 & 38 years & $\begin{array}{l}\text { Onabotulinu } \\
\text { mtoxinA }\end{array}$ & $\begin{array}{l}\text { Cervical } \\
\text { dystonia }\end{array}$ & $\begin{array}{c}\text { Single } \\
\text { injection } \\
200 \mathrm{U}\end{array}$ & $\begin{array}{c}\text { First } \\
\text { trimester } \\
2^{\text {ed }} \text { week }\end{array}$ & Alprazolam & $\begin{array}{l}\text { Healthy } \\
\text { term } \\
\text { newborn }\end{array}$ \\
\hline & & & Case 2 & 39 years & $\begin{array}{l}\text { Onabotulinu } \\
\text { mtoxinA }\end{array}$ & $\begin{array}{l}\text { Cervical } \\
\text { dystonia }\end{array}$ & $\begin{array}{c}\text { Single } \\
\text { injection } \\
500 \mathrm{U}\end{array}$ & $\begin{array}{c}\text { First } \\
\text { trimester } \\
\text { 4th week }\end{array}$ & $\begin{array}{c}\text { Benztropine } \\
\text {, C } \\
\text { clonazepam } \\
\text { and } \\
\text { fluoxetine } \\
\text { discontinue } \\
\text { d at } \\
\text { the } 6 \text { th } \\
\text { weeks }\end{array}$ & $\begin{array}{l}\text { Miscarriage } \\
\text { b } \\
\text { at } 10 \text { th } \\
\text { week }\end{array}$ \\
\hline \multirow{3}{*}{$\begin{array}{c}\text { De Oliveira } \\
\text { Monteiro } \\
(2006)[14]\end{array}$} & \multirow[t]{3}{*}{ Brazil } & \multirow[t]{3}{*}{ Case report } & 2 cases & & & & & & & \\
\hline & & & Case 1 & 34 years & $\begin{array}{l}\text { Onabotulinu } \\
\text { mtoxinA }\end{array}$ & $\begin{array}{l}\text { Cosmetic } \\
\text { facial } \\
\text { periorbital } \\
\text { lines }\end{array}$ & $\begin{array}{c}\text { Single } \\
\text { injection } \\
54 \mathrm{U}\end{array}$ & $\begin{array}{c}\text { First } \\
\text { trimester } \\
\text { 6th week }\end{array}$ & $\begin{array}{c}\text { Unmentione } \\
\mathrm{d}\end{array}$ & $\begin{array}{l}\text { Healthy } \\
\text { term } \\
\text { newborn }\end{array}$ \\
\hline & & & Case 2 & 37 years & $\begin{array}{c}\text { Onabotulinu } \\
\text { mtoxinA }\end{array}$ & $\begin{array}{c}\text { Cosmetic } \\
\text { facial } \\
\text { dynamic } \\
\text { lines;forehe } \\
\text { ad, glabelar, } \\
\text { and } \\
\text { periorbital }\end{array}$ & $\begin{array}{c}\text { Single } \\
\text { injection } \\
65 \mathrm{U}\end{array}$ & $\begin{array}{c}\text { First } \\
\text { trimester } \\
\text { 5th week }\end{array}$ & $\begin{array}{c}\text { Unmentione } \\
\mathrm{d}\end{array}$ & $\begin{array}{l}\text { Healthy } \\
\text { term } \\
\text { newborn }\end{array}$ \\
\hline $\begin{array}{c}\text { Morgan et } \\
\text { al. (2006) } \\
{[10]}\end{array}$ & USA & Survey & $\begin{array}{l}16 \text { cases }^{(19)^{c}}\end{array}$ & $\begin{array}{c}\text { Unmentione } \\
\mathrm{d}\end{array}$ & $\begin{array}{c}\text { Onabotulinu } \\
\text { mtoxinA }\end{array}$ & $\begin{array}{c}\text { Cervical } \\
\text { dystonia } \\
(9), \\
\text { Strabismus } \\
(2), \\
\text { Blepharospa } \\
\text { m } \\
(2), \\
\text { Limb } \\
\text { dystonia } \\
(1), \\
\text { Oromandibu } \\
\text { lar dystonia } \\
(1), \\
\text { Spasmodic } \\
\text { dysphonia } \\
(1)\end{array}$ & $\begin{array}{c}\text { Ranged } \\
\text { from } 1.25 \mathrm{U} \\
-300 \mathrm{U}\end{array}$ & $\begin{array}{c}\text { First } \\
\text { trimester } \\
(12), \\
\text { Second } \\
\text { trimester } \\
(1), \\
\text { Third } \\
\text { trimester } \\
(1), \\
\text { First, } \\
\text { second and } \\
\text { third } \\
\text { trimester } \\
\text { (1) } \mathbf{d}\end{array}$ & $\begin{array}{c}\text { Unmentione } \\
\mathrm{d}\end{array}$ & $\begin{array}{c}\text { Healthy } \\
\text { term } \\
\text { newborn } \\
(17), \\
\text { Miscarriage } \\
\text { e } \\
(1), \\
\text { Elective } \\
\text { abortion } \\
(1)\end{array}$ \\
\hline $\begin{array}{l}\text { Kuczkowski } \\
(2007)[15]\end{array}$ & USA & Case report & 1 case & 46 years & $\begin{array}{l}\text { Onabotulinu } \\
\text { mtoxinA }\end{array}$ & $\begin{array}{c}\text { Cosmetic } \\
\text { facial } \\
\text { injection }\end{array}$ & Unknown & $\begin{array}{c}\text { First } \\
\text { trimester }\end{array}$ & $\begin{array}{l}\text { Etomidate } \\
\text { (20 mg) } \\
\text { and } \\
\text { succinylchol } \\
\text { ine }(140 \mathrm{mg}) \\
\text { just before } \\
\text { Cesarean } \\
\text { section }\end{array}$ & $\begin{array}{c}\text { Unmentione } \\
\mathrm{d}\end{array}$ \\
\hline $\begin{array}{c}\text { Wataganara } \\
\text { et al. } \\
(2009)[16]\end{array}$ & Thailand & Case report & 1 case & 39 years & $\begin{array}{c}\text { Onabotulinu } \\
\text { mtoxinA }\end{array}$ & $\begin{array}{c}\text { Severe } \\
\text { Achalasia }\end{array}$ & $\begin{array}{c}\text { Single } \\
\text { injection } \\
80 \mathrm{U}\end{array}$ & $\begin{array}{l}\text { Third } \\
\text { trimester } \\
33 \text { week }\end{array}$ & $\begin{array}{c}\text { Total } \\
\text { parenteral } \\
\text { nutrition } \\
\text { was } \\
\text { started at } \\
\text { 33th } \\
\text { week }\end{array}$ & $\begin{array}{l}\text { Healthy } \\
\text { newborn } \\
36 \text { week }\end{array}$ \\
\hline
\end{tabular}




\begin{tabular}{|c|c|c|c|c|c|c|c|c|c|c|}
\hline $\begin{array}{l}\text { Li Yim and } \\
\text { Weir } \\
\text { (2010) [19] }\end{array}$ & UK & Case report & 1 case & 17 years & $\begin{array}{l}\text { Onabotulinu } \\
\text { mtoxinA }\end{array}$ & $\begin{array}{c}\text { Left } \\
\text { convergent } \\
\text { squint }\end{array}$ & $\begin{array}{c}\text { Single } \\
\text { injection } \\
2.5 \mathrm{U} / 0.1 \\
\mathrm{ml}\end{array}$ & $\begin{array}{c}\text { First } \\
\text { trimester }\end{array}$ & $\begin{array}{c}\text { Unmentione } \\
\mathrm{d}\end{array}$ & $\begin{array}{l}\text { Healthy } \\
\text { term } \\
\text { newborn } \\
9 \text { months }\end{array}$ \\
\hline $\begin{array}{c}\text { Aranda et } \\
\text { al. } \\
\text { (2012) [13] }\end{array}$ & Spain & Case report & 1 case & 41 years & $\begin{array}{l}\text { Onabotulinu } \\
\text { mtoxinA }\end{array}$ & $\begin{array}{l}\text { Cervical } \\
\text { dystonia }\end{array}$ & $\begin{array}{c}\text { Two } \\
\text { injections } \\
500 \mathrm{U} \\
\text { With } 250 \mathrm{U} \\
\text { in each }\end{array}$ & $\begin{array}{l}\text { Second and } \\
\text { third } \\
\text { trimester }\end{array}$ & $\begin{array}{c}\text { Unmentione } \\
\mathrm{d}\end{array}$ & $\begin{array}{c}\text { Healthy } \\
\text { term } \\
\text { newborn } \\
40 \text { th week }\end{array}$ \\
\hline $\begin{array}{l}\text { Robinson } \\
\text { and Grogan } \\
\text { (2014) [18] }\end{array}$ & USA & Case report & 1 case & 26 years & $\begin{array}{l}\text { Onabotulinu } \\
\text { mtoxinA }\end{array}$ & Migraine & $\begin{array}{c}\text { Single } \\
\text { injection } \\
71 \mathrm{U}\end{array}$ & $\begin{array}{c}\text { Second } \\
\text { trimester } \\
18 \text { th week }\end{array}$ & $\begin{array}{c}\text { Promethazin } \\
\text { e } \\
\text { metoclopra } \\
\text { mide, } \\
\text { acetaminop } \\
\text { hen, } \\
\text { butalbital, } \\
\text { caffeine, } \\
\text { isomethepte } \\
\text { ne } \\
\text { mucate, } \\
\text { Dichloralph } \\
\text { enazone }\end{array}$ & $\begin{array}{c}\text { Healthy } \\
\text { term } \\
\text { newborn } \$ \\
39 \text { week }\end{array}$ \\
\hline $\begin{array}{l}\text { Brin et al. } \\
\text { (2015) [7] }\end{array}$ & USA & Case series & $\begin{array}{c}232 \text { cases } \\
(237)\end{array}$ & $\begin{array}{c}\text { known ages } \\
\quad(146) \\
\\
<35 \text { years } \\
(76), \\
\geq 35 \\
\text { years } \\
(70)\end{array}$ & $\begin{array}{l}\text { Onabotulinu } \\
\text { mtoxinA }\end{array}$ & $\begin{array}{c}\text { Known } \\
\text { indications } \\
\text { (190) } \\
\text { Cosmetic } \\
\text { (96), } \\
\text { Movement } \\
\text { Disorders } \\
\text { (32), } \\
\text { Pain } \\
\text { disorders } \\
\text { (27), } \\
\text { Hyperhidros } \\
\text { is } \\
\text { (22), } \\
\text { Urological } \\
\text { disorders } \\
\text { (6), } \\
\text { Muscle } \\
\text { spasticity } \\
\text { (4), } \\
\text { GI disorders } \\
\text { (3) }\end{array}$ & $\begin{array}{c}\text { Known } \\
\text { doses } \\
(137) \\
\\
<50 \mathrm{U} \\
(55), \\
50 \mathrm{U} \text { to } \\
<100 \mathrm{U} \\
(20), \\
100 \mathrm{U} \text { to } \\
<150 \mathrm{U} \\
(26), \\
150 \mathrm{U} \text { to }< \\
200 \mathrm{U} \\
(12), \\
200 \mathrm{U} \text { to }> \\
400 \mathrm{U} \\
(24)\end{array}$ & $\begin{array}{c}\text { Known } \\
\text { times of } \\
\text { exposure } \\
(201) \\
0-3 \text { months } \\
\text { preconcepti } \\
\text { on (27), } \\
\text { First } \\
\text { trimester } \\
(166), \\
\text { Second } \\
\text { trimester } \\
(6), \\
\text { Third } \\
\text { trimester } \\
(2)\end{array}$ & $\begin{array}{c}\text { Unmentione } \\
\mathrm{d}\end{array}$ & $\begin{array}{c}\text { Healthy } \\
\text { newborn } \\
(167) \\
\text { Spontaneou } \\
\text { s abortion } \\
\text { (50), } \\
\text { Elective } \\
\text { abortion } \\
\text { (13), } \\
\text { Birth } \\
\text { defects } \\
\text { (5), } \\
\text { Birth } \\
\text { Complicatio } \\
\text { n } \\
\text { (1), } \\
\text { Adverse } \\
\text { event } \\
\text { (1) }\end{array}$ \\
\hline $\begin{array}{l}\text { Hooft et al. } \\
\text { (2015) [17] }\end{array}$ & USA & Case report & 1 Case & 23 years & $\begin{array}{l}\text { Onabotulinu } \\
\text { mtoxinA }\end{array}$ & Achalasia & $\begin{array}{c}\text { Single } \\
\text { injection } \\
100 \mathrm{U}\end{array}$ & $\begin{array}{c}\text { Second } \\
\text { trimester } \\
14 \text { th week }\end{array}$ & $\begin{array}{c}\text { Diltiazem } \\
\text {,imipramine } \\
\text { and } \\
\text { nitroglyceri } \\
\mathrm{n} \\
\text { discontinue } \\
\mathrm{d} \\
\text { at the 7th } \\
\text { week } \\
\text { of } \\
\text { pregnancy, } \\
\text { Cortisone } \\
\text { cream, } \\
\text { occasional } \\
\text { alcohol and } \\
\text { remote } \\
\text { social } \\
\text { smoking } \\
\text { exposure }\end{array}$ & $\begin{array}{l}\text { Healthy } \\
\text { term } \\
\text { newborn }\end{array}$ \\
\hline
\end{tabular}


a .Single case with four separate singleton pregnancies has been reported. b.Twin pregnancy with a history of one miscarriage before this pregnancy. c.One case carrying twins and another received injections during three separate singleton pregnancies. d. Received 1-3 injections (approximately $300 \mathrm{U}$ per treatment). e. Woman with a history of spontaneous abortion received BTX-A injection for cervical dystonia received one series of injections (approximately $300 \mathrm{U}$ ). f. The reasons for elective abortions were known in 9 cases: 5 personal/social, 1 fetal disorder, 1 high risk because of age, 1 blighted ovum, and 1 gestational sac with no embryo and one of the abortion involved the genetic abnormality (Down syndrome). g. Birth defects ; tracheoesophageal fistula/esophageal atresia (mother received $36 \mathrm{U}$ onabotulinumtoxinA, 3 days post conception for cosmetic facial wrinkles ), metatarsus adductus (mother received $100 \mathrm{U}$ onabotulinumtoxinA 15 days post-conception for migraine), laryngomalacia (mother received injection 1 week pre-conception and 2 weeks post-conception the exact dose wasn't reported for cosmetic facial wrinkles), tracheoesophageal fistula/esophageal atresia (mother received $36 \mathrm{U}$ onabotulinumtoxinA, 3 days post conception for cosmetic facial wrinkles), ventricular septal defect (mother received $100 \mathrm{U}$ onabotulinumtoxinA injection 37 days preconception for axillary hyperhidrosis) and innocent cardiac murmur ( family with history of cardiac murmur, mother received 8U, 12U, 14U onabotulinumtoxinA during first, second and trimester respectively for blepharospasm).

$\uparrow$ No findings of motor or developmental delay were detected in the 5 years follow-up. $\$$ The child has been followed for 6.5 years without any neuromuscular or developmental concerns

Table 2. Descriptive data of included studies for Botulism during pregnancy

\begin{tabular}{|c|c|c|c|c|c|c|c|c|c|c|}
\hline Study & $\begin{array}{c}\text { Study } \\
\text { location }\end{array}$ & $\begin{array}{l}\text { Type } \\
\text { of } \\
\text { Study }\end{array}$ & $\begin{array}{l}\text { No of } \\
\text { located } \\
\text { cases }\end{array}$ & $\begin{array}{c}\text { Women } \\
\text { age }\end{array}$ & $\begin{array}{c}\text { Type of } \\
\text { Botulism }\end{array}$ & $\begin{array}{l}\text { Botulinum } \\
\text { toxin } \\
\text { serotype }\end{array}$ & Case severity & $\begin{array}{c}\text { Gestational } \\
\text { age }\end{array}$ & $\begin{array}{l}\text { Pregnancy } \\
\text { complication } \\
\text { s }\end{array}$ & $\begin{array}{c}\text { Pregnancy } \\
\text { outcome }\end{array}$ \\
\hline $\begin{array}{l}\text { St Clair et } \\
\text { al., (1975) } \\
\text { [21] }\end{array}$ & USA & $\begin{array}{l}\text { Case } \\
\text { report }\end{array}$ & 1 case & 32 years & $\begin{array}{c}\text { Food-borne } \\
\text { botulism }\end{array}$ & Type A & $\begin{array}{l}\text { Required mechanical } \\
\text { ventilation; treated } \\
\text { with antitoxin }\end{array}$ & $\begin{array}{l}\text { Third } \\
\text { trimester } \\
34 \text { week }\end{array}$ & $\begin{array}{l}\text { Partial } \\
\text { placental } \\
\text { abruption and } \\
\text { precipitous } \\
\text { delivery }\end{array}$ & $\begin{array}{c}\text { Preterm infant }{ }^{1} \\
34 \text { week, } \\
\text { intraventricular } \\
\text { hemorrhage after } \\
\text { birth, } \\
\text { hydrocephalus } \\
\text { several months } \\
\text { later, at } 20 \\
\text { months } \\
\text { developmental } \\
\text { delay and } \\
\text { blindness }\end{array}$ \\
\hline $\begin{array}{l}\text { CDC } \\
\text { MMWR, } \\
\text { (1995) [27] }\end{array}$ & USA & $\begin{array}{l}\text { Case } \\
\text { report }\end{array}$ & 1 case & 30 years & $\begin{array}{c}\text { Wound } \\
\text { botulism }\end{array}$ & Type A & $\begin{array}{l}\text { Ophthalmoplegia, } \\
\text { proximal paralysis of } \\
\text { arms and legs, } \\
\text { required mechanical } \\
\text { ventilation, treated } \\
\text { with antitoxin }\end{array}$ & Unmentioned & Unmentioned & $\begin{array}{c}\text { Preterm infant } \\
34 \text { weeks } \\
\text { kept in intensive } \\
\text { care for several } \\
\text { weeks }\end{array}$ \\
\hline $\begin{array}{l}\text { Polo et al } \\
\text { (1996) [22] }\end{array}$ & Spain & $\begin{array}{l}\text { Case } \\
\text { report }\end{array}$ & 1 case & 37 years & $\begin{array}{c}\text { Food-borne } \\
\text { botulism }\end{array}$ & $\begin{array}{l}\text { Couldn't } \\
\text { be } \\
\text { identified }\end{array}$ & $\begin{array}{l}\text { Tetraparesis and } \\
\text { required mechanical } \\
\text { ventilation, treated } \\
\text { with antitoxin }\end{array}$ & $\begin{array}{l}\text { Second } \\
\text { trimester } \\
23 \text { week }\end{array}$ & $\begin{array}{c}\text { None } \\
\text { Spontaneous } \\
\text { delivery at } 33 \\
\text { week }\end{array}$ & $\begin{array}{c}\text { Preterm infant } \\
33 \text { weeks } \\
\text { without any fetal } \\
\text { complications or } \\
\text { congenital } \\
\text { anomalies }\end{array}$ \\
\hline $\begin{array}{l}\text { Robin et al. } \\
\text { (1996) [23] }\end{array}$ & USA & $\begin{array}{l}\text { Case } \\
\text { report }\end{array}$ & 1 case & 24 years & $\begin{array}{c}\text { Food-borne } \\
\text { botulism }\end{array}$ & $\begin{array}{c}\text { Type A } \\
\text { and type } \mathrm{E}\end{array}$ & $\begin{array}{l}\text { forced vital capacity } \\
\text { was } 76 \% \text { of the } \\
\text { predicted value, } \\
\text { treated with antitoxin }\end{array}$ & $\begin{array}{l}\text { Second } \\
\text { trimester } \\
16 \text { weeks }\end{array}$ & None & $\begin{array}{l}\text { Health term } \\
\text { newborn } \\
42 \text { week }\end{array}$ \\
\hline $\begin{array}{l}\text { Götz et al. } \\
\text { (2002) [24] }\end{array}$ & Germany & $\begin{array}{l}\text { Case } \\
\text { report }\end{array}$ & 1 case & 37 years & $\begin{array}{l}\text { Food-borne } \\
\text { botulism }\end{array}$ & Type A & $\begin{array}{l}\text { Tetraparesis, required } \\
\text { mechanical } \\
\text { ventilation, treated } \\
\text { with antitoxin }\end{array}$ & Unmentioned & $\begin{array}{l}\text { Spontaneous } \\
\text { abortion }\end{array}$ & $\begin{array}{l}\text { Miscarriage } \\
\text { six days after } \\
\text { beginning of } \\
\text { symptoms }\end{array}$ \\
\hline $\begin{array}{l}\text { Magri et } \\
\text { al.(2006) } \\
{[25]}\end{array}$ & France & $\begin{array}{l}\text { Case } \\
\text { report }\end{array}$ & 1 case & 28 years & $\begin{array}{c}\text { Food-borne } \\
\text { botulism }\end{array}$ & Type B & $\begin{array}{l}\text { Required intubation } \\
\text { for respiratory } \\
\text { deterioration, not } \\
\text { treated with antitoxin }\end{array}$ & $\begin{array}{l}\text { Second } \\
\text { trimester } \\
26 \text { weeks }\end{array}$ & None & $\begin{array}{l}\text { Healthy term } \\
\text { newborn } \\
40 \text { week }\end{array}$ \\
\hline $\begin{array}{l}\text { Morrison et } \\
\text { al. }(2006) \\
\text { [26] }\end{array}$ & UK & $\begin{array}{l}\text { Case } \\
\text { report }\end{array}$ & 1 case & 23 years & $\begin{array}{c}\text { Wound } \\
\text { botulism }\end{array}$ & Type A & $\begin{array}{l}\text { Required full mech } \\
\text { ventilation } 24 \text { hours, } \\
\text { then ventilation assist } \\
30 \text { days; treated with } \\
\text { equine antitoxin }\end{array}$ & $\begin{array}{l}\text { Third } \\
\text { trimester } \\
36 \text { weeks }\end{array}$ & $\begin{array}{l}\text { Urgent } \\
\text { Caesarean } \\
\text { delivery in } \\
\text { view of } \\
\text { deteriorating }\end{array}$ & $\begin{array}{c}\text { Preterm infant }{ }^{4} \\
36 \text { week } \\
\text { required } \\
\text { treatment for a } \\
\text { respiratory tract }\end{array}$ \\
\hline
\end{tabular}




\begin{tabular}{|l|l|l|l|l|l|l|l|l|l|}
\hline & & & & & & $\begin{array}{c}\text { infection and } \\
\text { drug withdrawal }\end{array}$ \\
\hline $\begin{array}{c}\text { Leclair et al. } \\
\text { (2013) [20] }\end{array}$ & Canada & $\begin{array}{c}\text { Case } \\
\text { series }\end{array}$ & 3 cases & $\begin{array}{c}\text { unmention } \\
\text { ned }\end{array}$ & $\begin{array}{c}\text { Food-borne } \\
\text { botulism }\end{array}$ & $\begin{array}{c}\text { Type E } \\
2 \text { cases } \\
\text { Type B } \\
1 \text { case }\end{array}$ & $\begin{array}{c}\text { Persistent toxemia for } \\
\text { 10 days during the } \\
\text { first trimester } \\
1 \text { case } \\
\text { Unmentioned } \\
2 \text { cases }\end{array}$ & $\begin{array}{c}\text { First trimester } \\
1 \text { case }\end{array}$ & $\begin{array}{c}\text { None } \\
\text { Healthy infants } \\
\text { in 3 cases } \\
\text { exact times were } \\
\text { unmentioned }\end{array}$ \\
\hline
\end{tabular}

1.No detectable toxins in infant serum $4 \mathrm{~h}$ after birth. 2 . The growth and development were normal during infant first year of life. 3. The woman was pregnant during the use of IUD for birth control. 4. The infant required no treatment for botulism.

Figure 1. PRISMA flow diagram

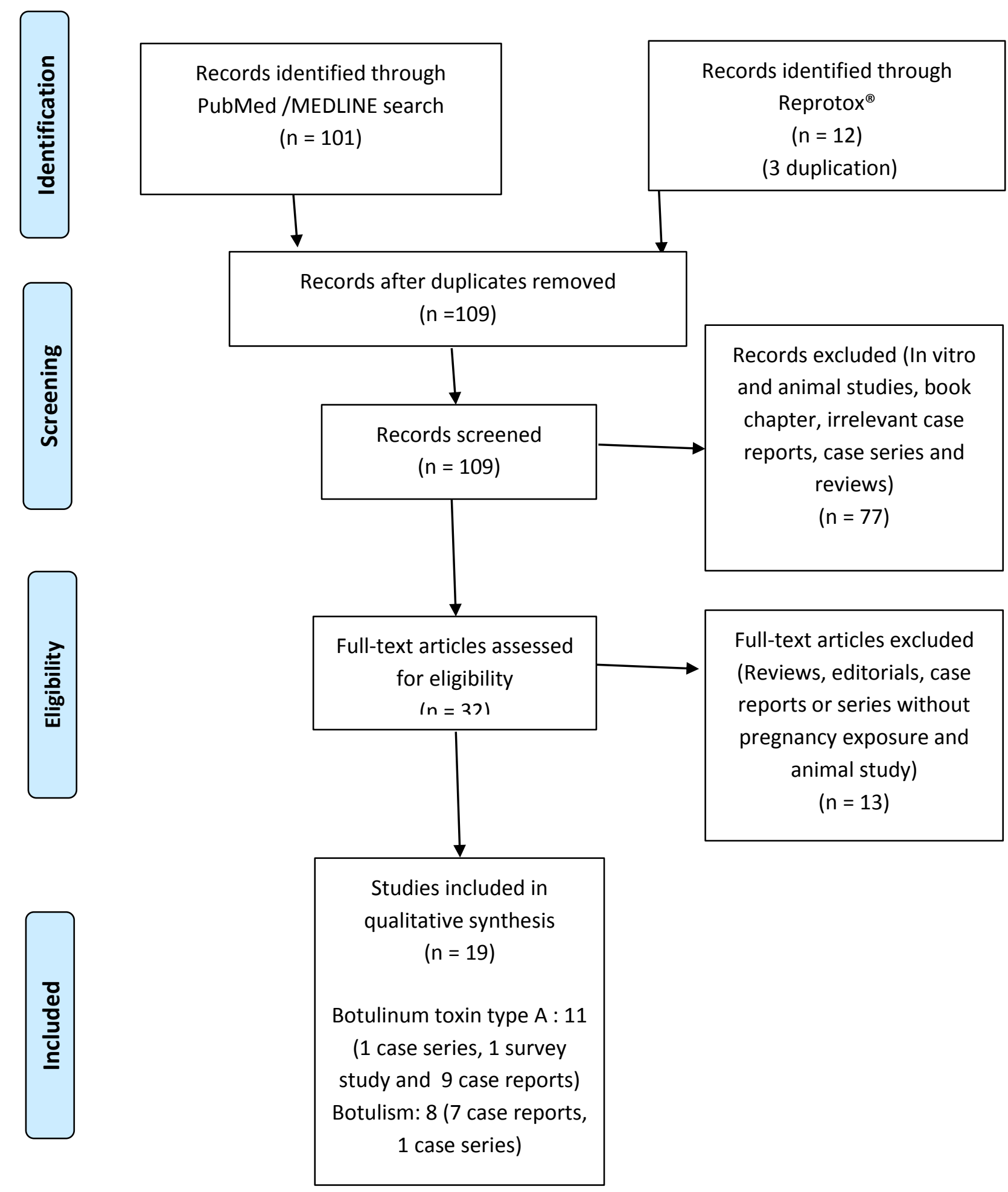




\section{Therapeutic exposure for BTX-A during pregnancy}

\subsection{Review of the case series regarding therapeutic BTX-A administration during pregnancy}

A retrospective review by Brin et al. [7] examined Allergan Global Safety Database between years 1990 and 2013. The database includes prospective (pregnancies recorded before the outcome was known) or retrospective (pregnancies recorded after the outcome was known) cases. Among 574 reported cases, 232 eligible women (237 infants) with known outcome have been included in Brin et al. [7] study. In the prospective part; 137 pregnancies (139 fetuses) ended with 110 live births (79.1\%), four of which presented with abnormal outcomes; three birth defects 11 major fetal defect; ventricular septal defect, 2 minor fetal malformations; metatarsus adductus and innocent cardiac murmur] and one birth complication [Horner syndrome] . The prevalence of overall congenital malformations was $2.7 \%$ (3/110; 95\% CI, 0.6-8.0\%) and major malformations were $0.9 \%(1 / 110 ; 95 \% \mathrm{CI}, 0.02-5.1)$ which was comparable with the baseline rates of 3-5\% [8]. In the retrospective part of Brin et al. [7] study; 95 pregnancies (98 fetuses) ended with 64 live births, three of which were with an abnormal outcome [1 major fetal defect; tracheoesophageal fistula/esophageal atresia, 1 minor fetal malformation; laryngomalacia, and 1 significant adverse event; benign brain tumor identified at 13 months]. The previously identified malformations presented no distinct pattern [7].

The observed prevalence rates of spontaneous abortion and elective termination of pregnancy in the prospective part of this study were $15.1 \%$ (21/139; 95\% CI, 9.4-23.1\%) and 5.8\% (8/139; 95\% CI, 2.5$11.3 \%$ ). These rates were comparable to that of the general population in the United States for spontaneous abortion and elective termination of pregnancy $17.0 \%$ and $18.4 \%$ respectively [9]. However, in the retrospective part, the rates of fetal loss $(34.7 \%)$ and elective termination rates $(14.7 \%)$ were much higher than the background rates [9]. Brin et al [7] mentioned that in the total cases with known ages, indication, time of fetal exposure and dose of BTX-A; nearly half of them 70/146 (47.9\%) were of advanced age ( $\geq 35$ years), and 96/190 (50.5\%) used for cosmetic purposes, most 166/201 (82.6\%)of fetal exposure occurred during the first trimester and the majority 55/137(40.1\%) received $(<50 \mathrm{U})$ of onabotulinumtoxinA [ Table 1 ].

\subsection{Review of the physician survey regarding therapeutic BTX-A administration during pregnancy}

Morgan et al. [10] surveyed 900 physicians whether they administered BTX-A to pregnant women and if they had the information regarding the pregnancy outcome. Among the 396 physicians who responded to the survey, 12 had reported administering BTX-A to 16 women during their pregnancies. Most of the pregnant women (12) had received the injection during the first trimester. Interestingly, one pregnant woman was injected with BTX-A in three different pregnancies during different trimesters [received 1-3 injections approximately $300 \mathrm{U}$ per treatment]. The injected BTX-A dose ranged from 1.25 to $300 \mathrm{U}$ of onabotulinumtoxinA. The reported indications were cervical dystonia ( 9 patients), strabismus (2), blepharospasm (2), limb dystonia (1), oromandibular dystonia (1), and spasmodic dysphonia (1). A total of 19 fetuses from 18 pregnancies (one twin pregnancy) were exposed to BTX-A in utero. Seventeen infants were born full term without complications and did not receive any special care after birth while one pregnancy ended with a miscarriage [history of miscarriage received approximately $300 \mathrm{U}$ for cervical dystonia one injection ] another was electively terminated [10].

\subsection{Review of the case reports regarding therapeutic BTX-A administration during pregnancy}

Newman et al. [11] reported a single patient who had four uncomplicated full-term pregnancies while receiving regular BTX-A treatments, three months before pregnancy, during first, second and third trimesters in doses ranging from 600-1200 U, for the treatment of cervical dystonia. She delivered 4 healthy children without any congenital malformations and with no findings of cognitive or motor developmental delay during the follow-up period 
which was approximately five years for the oldest child. Bodkin et al. [12] and Aranda et al. [13] reported three pregnant women with cervical dystonia who received BTX-A injection during pregnancy. Two of these pregnancies resulted in healthy term babies, and one resulted in a miscarriage. It is, however, difficult to determine whether there was an association between BTX-A exposure and miscarriage since the 39-yearold mother had a twin gestation with prior history of miscarriage and used benztropine, clonazepam, and fluoxetine beside BTX-A during her pregnancy [12]. De Oliveira Monteiro [14] reported two patients who experienced uncomplicated full-term pregnancies after receiving BTX-A injection (54 $\mathrm{U}$ and $65 \mathrm{U}$ ) in the first trimesters for cosmetic purpose. Both of them delivered healthy term babies by cesarean section. Kuczkowski [15] reported another 46-year pregnant women with exposure to BTX-A for cosmetic purposes. Because the mother had a worsening severe pregnancy-induced hypertension and HELLP syndrome, she required cesarean section under general anesthesia during which she failed to respond to the train-of-four test which is used for the detection of patients' recovery from neuromuscular blockade. Later, it was ascertained that multiple bilateral facial administrations of BTX-A were done due to the cosmetic reasons in the early weeks of gestation. The outcome of the infant, however, was unmentioned. Wataganara et al. [16] and Hooft et al. [17] described two pregnant women who received BTX-A injection into the lower esophageal sphincter for achalasia. The former described a 39-year-old pregnant woman at the 33 weeks of gestation with severe esophageal achalasia, weight loss, severe malnutrition, dehydration and fetal growth restriction (1400 g/10th percentile) who received a total of $80 \mathrm{U}$ of BTX-A without any complications. Improvement in the maternal weight was detected after BTX-A injection; fetal weight was $1600 \mathrm{~g}$ (10th percentile) at the 35th week and the mother gained a total of $5 \mathrm{~kg}$ at 36th week of pregnancy. The mother gave birth to a $1800 \mathrm{~g}$ (10th percentile) female infant via cesarean section and the baby displayed no signs of neuromuscular blockade after close observation for 5 days [16]. The latter report [17] described a 23-yearold female with type III achalasia who was unresponsive to medical management. She was reported to have a serious drop in her body mass index $(\mathrm{BMI}=15.6)$ and treated for dehydration in the emergency department twice during her pregnancy. She was successfully managed by administering BTX-A with a total dose of $400 \mathrm{U}$ at the 14th week of gestation. The patient reported a significant relief for her symptoms such as dysphagia, pain and regurgitation. She was reported to gain approximately $4.5 \mathrm{~kg}$ (BMI increased to 18.2) and gave birth to a healthy female infant at term. No complications were observed [17]. Robinson and Grogan [18] reported 26-year-old female patient with 10-years history of migraine who started receiving regular BTX-A injections 22 months before pregnancy. BTX-A injections, however, were withheld because of pregnancy. After the failure of the standard headache treatments by the 18 weeks of pregnancy she gave consent to restart BTX-A injections. She received a total dose of $71 \mathrm{U}$ and delivered a full-term healthy girl who showed no signs of developmental or neuromuscular problems at the age of 6.5 [18]. The last reported case was described by Li Yim, and Weir [19] who was 17-year-old female received BTX-A injection (2.5 units $/ 0.1 \mathrm{ml}$ ) in the left medial rectus muscle for the treatment of left convergent squint and 20 prism diopter for near and distance vision. Six months later she received another BTX-A injection due to the increase in her convergent squint again. Three days after the injection she discovered that she was pregnant. The course of the pregnancy was normal and she delivered a healthy baby (Table 1).

\section{Botulism during pregnancy}

\subsection{Review of the cases reporting outcomes of pregnant women with foodborne and wound botulism}

Six studies of food-borne botulism have been retrieved; Leclair et al. [20] reported case series for foodborne botulism between (1985 - 2005), where three of the reported cases were pregnant women, two of them exposed to type $\mathrm{E}$ and the third one to type B botulinum toxin serotypes, all of the 3 women delivered healthy infants although one of them had persistent toxemia for $>10$ days during the first trimester of pregnancy. St Clair et al [21] reported a case for 32-year woman suffered from botulism (type A) approximately at the 34th week of gestation. The woman required ventilatory assistance and treated with 
antitoxin. She had partial placental abruption and precipitous delivery of a preterm male infant (34 weeks) where there was no detectable toxin in infant serum 4 hrs after birth. The infant suffered from intraventricular hemorrhage after birth, hydrocephalus was noted several months later and at 20 months of age, he showed developmental delay and blindness [21]. Polo et al. [22] reported a 37-years pregnant woman with botulism at the 23rd week of her gestation, botulinum toxin serotypes couldn't be identified, the woman experienced tetraparesis, required ventilatory assistance and treated with an antitoxin, at 33 weeks she had a spontaneous delivery without any fetal complications or congenital anomalies. Robin et al. [23] reported a botulism case of a 24-year female with type A and E at 16 weeks of gestation. Her forced vital capacity was $76 \%$ of the predicted value and treated with antitoxin. The patient delivered a healthy boy at 42 weeks of pregnancy of whose growth and development was normal during his first year of life. Götz et al. [24] described a 37-year woman with type A serotype botulism who suffered from respiratory failure and tetraparesis. Mechanical ventilation was performed and antitoxin was given, the woman was pregnant during the use of an IUD for birth control, exact week of gestation was not mentioned, the pregnancy ended with spontaneous abortion six days after the beginning of the symptoms. Magri et al. [25] reported 28-years patient with botulism (type B), during the 26th week of gestation who required intubation for respiratory deterioration, but did not receive antitoxin. At the 40th week, she spontaneously delivered healthy fetus [Table 2].

Two studies of wound botulism were also retrieved in our search.; Morrison et al. [26] described 23years women experienced type A serotype botulism due to intravenous drug abuse at 36 weeks of gestation who, required full mechanical ventilation for $24 \mathrm{hrs}$ and ventilatory assistance for 30 days. She was; treated with equine antitoxin as a result of her deteriorating ventilatory function. An urgent Caesarean section decision was made and a live female infant was delivered at the 36th week who required treatment for a respiratory tract infection and drug withdrawal. However, no specific treatment was needed with respect to her mother's diagnosis. In a report by the Centers for Disease Control and Prevention (CDC) 30-year-old pregnant woman hospitalized for mechanical ventilation because of wound botulism (type A) was described. She gave birth by cesarean section at 34 weeks of gestation and, her baby was reported to stay in intensive care for several weeks, however, details were not provided [27] [Table 2].

\section{Discussion}

In this systematic review, we were only able to retrieve case series and case reports but no controlled epidemiological studies regarding the outcomes after therapeutic administration of BTX-A during pregnancy. Considering limitations of the data, our review suggests that BTX-A exposure during pregnancy does not seem to be associated with increased rates or a distinct pattern of major congenital malformations. The rates of fetal loss in prospective data were much lower than the retrospective data and comparable to that of the general population. No increased rates of prematurity or low birth weight were identified. Since molecules less than $500 \mathrm{Da}$ have incomplete transfer [28], BTX-A is a large-molecule (150.000 D), therefore, it is not possible to cross the placental barrier [29]

According to the animal studies no adverse effects on fetal development were observed when pregnant rats received single BTX-A intramuscular injections $(1,4$, or $16 \mathrm{U} / \mathrm{kg}$ ) at the three critical periods of development (prior to implantation, implantation, or organogenesis). The highest no effect dose for developmental toxicity ( $16 \mathrm{U} / \mathrm{kg}$ ) is 2 times the maximum recommended human dose based on Units/kg [2]. While a reductions in fetal body weight and decreased fetal skeletal ossification observed in pregnant rats or mice that were observed after two injections during the organogenesis period (days 5 and 13 of gestation) of

BTX-A $(4,8$, or $16 \mathrm{U} / \mathrm{kg})$ with the highest two doses. The no-effect dose for developmental toxicity (4 $\mathrm{U} / \mathrm{kg}$ ) is approximately equal to the maximum recommended human dose of 400 Units on a body weight 
basis (U/kg) [2]. In pregnant rabbits there appear to be no transfer of BTX-A toxin to fetal blood of poisoned parents after intravenous injection [30].

In the limited reviewed cases, in which therapeutic exposure to BTX-A occurred in the late pregnancy or the mother was diagnosed with botulism, suggested no acute adverse effects of BTX-A on fetal movements.

Wataganara et al. [16] reported that sonographic examinations did not show any evidence of botulinum toxicity in the fetus after the mother received onabotulinumtoxinA (80U) injection at 33 weeks of gestation and no signs of neuromuscular blockade after 5 days of monitoring after delivery. Robinson and Grogan [18] reported normal fetal movements were noted throughout the pregnancy after the mother received a total dose of $71 \mathrm{U}$ onabotulinumtoxinA at the 18 weeks of pregnancy. Reassuringly the 10 pregnant women who exposed to botulism during pregnancy, none of the 9 live births appeared to require treatment for botulism [20-27]. Even in the in three fetuses with perinatal complications in women with botulism [21, 26, 27]. These complications, however, may be associated with the complicated birth [21] or medical condition of their mother [26].

Data regarding the possible long-term effects of in-utero BTX-A exposure on children were very limited. Nevertheless, no long-term complications were reported. Robinson and Grogan [18] reported the longest follow-up (6.5 years) of a child with no neuromuscular or developmental concerns. Newman et al. [11] reported no complications among four exposed children during their follow-up, which was approximately 5 years for the oldest child. Aranda et al. [13] described a normally developing infant during the first 7 th months of life. Robin et al. [23] reported a healthy boy where his growth and development were normal during his first year of life.

The main limitation of our review is the inclusion of uncontrolled studies with a small number of cases which precludes a definitive conclusion on the safety of BTX-A treatment during pregnancy. Nevertheless, we believe that this review still offers value to the clinicians who counsel pregnant women with inadvertent BTX-A use during pregnancy or who considers BTX-A treatment for pregnant or reproductive-age women for a clinical indication.

\section{Conclusion}

Although data is limited to case series and case reports this review suggests that therapeutic exposure to BTX-A or botulism during pregnancy has not been associated with major congenital malformations. Prospective data suggested no increase in rates of miscarriage and elective termination of pregnancy whereas retrospective data, which is prone to recall bias, indicated the exact opposite. No increased rates of prematurity or low birth rate were detected. Healthy births are even reported in a limited number of pregnancies with severe food-borne or wound botulism. There is apparently no reason to terminate a pregnancy after an inadvertent BTX-A exposure, however, controlled epidemiological studies are needed to refute or support our interpretation.

\section{Disclosure statement}

The authors declare no conflict of interest.

\section{Funding}

This study had no funding source.

\section{References}

[1] Jabbari B. History of Botulinum Toxin Treatment in Movement Disorders. Tremor Other Hyperkinet Mov (N Y). 2016:28;6:394. eCollection 2016. PMID: 27917308 .

[2] BOTOX® [package insert]. Irvine, CA: Allergan, Inc.? 2015. http://www.allergan.com/assets/pdf/botox_pi.pdf [accessed 4 January 2016]. 
[3] Awan KH. The therapeutic usage of botulinum toxin (Botox) in non-cosmetic head and neck conditions - An evidence based review. Saudi Pharm J. 2017;25(1):18-24. PMID: 28223858.

[4] Barnes MP, Best D, Kidd L, Roberts B, Stark S, Weeks P and Whitaker J. The use of botulinum toxin type-B in the treatment of patients who have become unresponsive to botulinum toxin type-A - initial experiences. Eur J Neurol 2005;12:947-55. PMID: 16324088.

[5] Finer LB, Zolna MR. Unintended pregnancy in the United States: incidence and disparities, 2006. Contraception 2011; 84:478-85. PMID: 22018121.

[6] Moher D, Liberati A, Tetzlaff J, Altman DG; The PRISMA Group. Preferred Reporting Items for Systematic Reviews and Meta-Analyses: The PRISMA Statement. PLoS Med 6(7): e1000097. PMID: 19621072.

[7] Brin MF, Kirby RS, Slavotinek A, Miller-Messana MA et al. Pregnancy outcomes following exposure to onabotulinumtoxinA. Pharmacoepidemiol Drug Saf. 2016;25(2):179-87. PMID: 26635276.

[8] Centers for Disease Control and Prevention (CDC). Update on overall prevalence of major birth defects-- Atlanta,Georgia, 1978-2005. MMWR Morb Mortal Wkly Rep 2008; 57: 1-5. PMID: 18185492.

[9] Ventura SJ, Curtin SC, Abma JC, Henshaw SK. Estimated pregnancy rates and rates of pregnancy outcomes for the United States, 1990-2008. Natl Vital Stat Rep 2012; 60: 1-21. PMID: 22970648.

[10] Morgan JC, Iyer SS, Moser ET, Singer C and Sethi KD. Botulinum toxin A during pregnancy: a survey of treating physicians. $J$ Neurol Neurosurg Psychiatry. 2006;77(1):117-119. PMID: 16361610.

[11] Newman WJ, Davis TL, Padaliya BB, Covington CD, Gill CE, et al., Botulinum toxin type a therapy during pregnancy. Mov Disord. 2004;19(11):1384-5. PMID:15389988.

[12] Bodkin C, Maurer K, Wszolek Z. Re: Botulinum toxin type A therapy during pregnancy. Mov Disord. 2005;20(8):1081-2; author reply 1082. PMID: 16007618

[13] Aranda MA, Herranz A, del Val J, Bellido S, García-Ruiz P. Botulinum toxin A during pregnancy, still a debate. Eur J Neurol. 2012;19(8):e81-2. PMID: 22805647.

[14] De Oliveira Monteiro E. Botulinum toxin and pregnancy. Skinmed. 2006; 5(6):308. PMID: 17086003.

[15] Kuczkowski KM. Anesthetic implications of botulinum toxin type A (Botox) injections for the treatment of 'the aging face' in the parturient. Acta Anaesthesiol Scand. 2007;51(4):515-6. PMID: 17378795.

[16] Wataganara T, Leelakusolvong S, Sunsaneevithayakul P and Vantanasiri C.Treatment of severe achalasia during pregnancy with esophagoscopic injection of botulinum toxin A: a case report. J Perinatol. 2009;29(9):637-9. PMID: 19710656.

[17] Hooft N, Schmidt ES, and Bremner RM . Achalasia in Pregnancy: Botulinum Toxin A Injection of Lower Esophageal Sphincter. Case Rep Surg. 2015;2015:328970. PMID: 26229704.

[18] Robinson MA, and Grogan PM. OnabotulinumtoxinA Successfully Used as Migraine Prophylaxis During Pregnancy: A Case Report. Mil Med. 2014;179(6):e703-4. PMID: 24902141

[19] Li Yim JFT, and Weir CR. Botulinum Toxin and Pregnancy- A Cautionary Tale. Strabismus. 2010;18(2):65-6. PMID: 20521881.

[20] Leclair D, Fung J, Isaac-Renton JL, Proulx JF, May-Hadford J et al. Foodborne botulism in Canada, 1985-2005. Emerg Infect Dis. 2013;19(6):961-8. PMID: 23735780.

[21] St Clair EH, DiLiberti JH and O'Brien ML. Letter: observations of an infant born to a mother with botulism. J Pediatr. 1975;87(4):658. PMID: 1159598.

[22] Polo JM, Martin J, and Berciano, “Botulism and pregnancy. Lancet. 1996;20;348(9021): 195. PMID: 8684170. 
[23] Robin L, Herman D and Redett R. Botulism in a pregnant women. N Engl J Med. 1996; 12;335(11):823-4. PMID: 8778597.

[24] Götz J, Carlsson J, Schipmann R , Rohde M, Sorges E, Manz F, Tebbe U.37-year-old patient with eye muscle paralysis and rapidly progressing respiratory insufficiency.[Article in German]. Internist (Berl). 2002 ; 43(4): 548-553. PMID:12053411.

[25] Magri K, Bresson V and Barbier C. Botulism and pregnancy [Article in French]. J Gynecol Obstet Biol Reprod (Paris). 2006;35(6):624-6. PMID: 17003751.

[26] Morrison GA, Lang C and Huda S. Botulism in a pregnant intravenous drug abuser. Anaesthesia. 2006 ;61(1):57-60. PMID: 16409344.

[27] Centers for Disease Control and Prevention (CDC). Wound botulism--California, 1995. MMWR Morb Mortal Wkly Rep. 1995;;44(48):889-92. PMID: 7476845.

[28] Pacifici GM and Nottoli R. Placental transfer of drugs administered to the mother. Clin Pharmacokinet. 1995;28(3):235-69. PMID: 7758253.

[29] https://chem.nlm.nih.gov/chemidplus/rn/93384-43-1.

[30] Hildebrand GJ, Lamana C, Heckly RJ. Distribution and particle size of type A botulinum toxin in body fluids of intravenously injected rabbits. Proc Soc Exp Biol Med 1961; 107: 284-289. DOI: https://doi.org/10.3181/00379727-107-26604 